hep-th/0012218

CALT-68-2311

CITUSC/00-066

\title{
How Noncommutative Gauge Theories Couple to Gravity
}

\author{
Yuji Okawa and Hirosi Ooguri \\ California Institute of Technology 452-48, Pasadena, CA 91125 \\ and \\ CIT-USC Center for Theoretical Physics, Los Angeles, CA 90089 \\ okawa, ooguri@theory.caltech.edu
}

\begin{abstract}
We study coupling of noncommutative gauge theories on branes to closed string in the bulk. We derive an expression for the gauge theory operator dual to the bulk graviton, both in bosonic string theory and superstring theory. In either case, we find that the coupling is different from what was expected in the literature when the graviton is polarized in the noncommutative directions. In the case of superstring, the expression for the energy-momentum tensor is consistent with the way the bulk metric appears in the action for the noncommutative gauge theory. We also clarify some aspects of the correspondence between operators in the gauge theory and boundary conditions in the dual gravitational description.
\end{abstract}




\section{Introduction}

Noncommutative gauge theories can be realized as the zero slope limit of open string theory on branes in a background of strong NS-NS two-form field $B_{i j}[1-11]$. It was pointed out in $[12,13]$ that, for each local observable in a gauge theory in commutative space, one can define an observable in the noncommutative version of the theory. The construction is done in momentum space and involves an open Wilson line [14] whose end-points are separated by a vector $l^{i}$ defined by

$$
l^{i}=k_{j} \theta^{j i}
$$

where $k_{i}$ is the momentum carried by the operator, and $\theta^{i j}$ is the noncommutative parameter,

$$
\left[x^{i}, x^{j}\right]=-i \theta^{i j} .
$$

The condition (1) is necessary in order to maintain gauge invariance. Various aspects of the open Wilson line were discussed in [15 - 19]. Open Wilson lines make an overcomplete set of gauge invariant observables. An open Wilson line of any shape is gauge invariant as long as its end-points are separated by $l^{i}$. We can also insert operators in various ways along the line.

In fact, there is a canonical prescription to define gauge invariant observables in noncommutative theories corresponding to a large class of operators in commutative theories. These are operators which describe the coupling of the gauge theory on the brane to closed string states in the bulk. In this paper, we find precise expressions of these operators in noncommutative theories by studying disk amplitudes of one closed string and arbitrary number of open strings and by taking the zero slope limit. Disk amplitudes with a closed string state in the presence of a constant $B$ field were discussed in $[20,21]$, and it was found that the amplitudes involve the so-called generalized star products. $^{1}$ In [25], it was shown that these products also appear if one considers an open Wilson line which connects $x$ and $x+l$ by a straight line and expands it in powers of gauge fields. Combining these observations, it was anticipated that the open Wilson lines play some role in constructing gauge theory operators coupled to closed string states. The link between generalized star products and open Wilson lines has been investigated further in [26 - 30].

In this paper, we set up our computation so that we can determine the gauge theory operators that couple to arbitrary excited closed string states. We show that a basic ingredient of such operators is a straight open Wilson line of the form,

$$
\int d x *\left[e^{i k x} \exp \left(i \int_{0}^{1} d \tau l^{i} A_{i}(x+l \tau)+y_{\alpha} \Phi^{\alpha}(x+l \tau)\right)\right],
$$

\footnotetext{
${ }^{1} \mathrm{~A}$ similar structure also appear in one-loop amplitudes of these theories [22 - 24].
} 
where $x$ are the coordinates on the brane, $A_{i}$ is the gauge field in the noncommutative directions, $\Phi^{\alpha}$ is the scalar field describing the transverse positions of the branes, and $*[\cdots]$ means that we take the standard star product in the expansion of the expression in [...] in powers of $A_{i}$ and $\Phi^{\alpha}$. The path-ordered exponential is implicit in this expression and throughout the rest of the paper. The vector $l^{i}$ is defined by (1) and $y_{\alpha}$ is given by

$$
y_{\alpha}=2 \pi \alpha^{\prime} k_{\alpha},
$$

where $k_{\alpha}$ is the momentum in the directions transverse to the brane. Because of the closed string on-shell condition, the Wilson line can couple to a closed string only when $l^{i}$ and $y_{\alpha}$ satisfy the constraint

$$
G_{i j} l^{i} l^{j}+g^{\alpha \beta} y_{\alpha} y_{\beta}=0
$$

where $G_{i j}$ is the open string metric in the noncommutative directions and $g_{\alpha \beta}$ is the closed string metric in the directions transverse to the brane.

The condition (5) is independent of the amount of closed string excitations. For coupling with excited states, we need to insert additional gauge theory operators along the Wilson line (3). We describe a systematic procedure to determine these additional operators and the way they are inserted along the Wilson line.

We derive an explicit expression for the operator coupled to the bulk graviton, i.e., the energy-momentum tensor. In the case of bosonic string, the structure of the operator suggests that the leading coupling between the bulk metric and the gauge fields is through the curvature of the metric. In the case of superstring, the leading coupling to the curvature is absent. We then go to the next leading order in the $\alpha^{\prime}$ expansion of the disk amplitude and derive the energy-momentum tensor. We find that the resulting expression agrees, in the limit of zero momentum, with the variation of the action of the noncommutative gauge theory [10] with respect to the bulk metric. In both bosonic and supersymmetic cases, we find that the coupling between the gauge fields and the bulk metric is different from what was expected in the literature when the graviton is polarized in the noncommutative directions.

A general structure of the gauge theory operators fits well with the proposed gravity duals of the noncommutative theories at large $N[31,32]$. In a gauge theory in commutative space, it is natural to classify operators into irreducible representations of global symmetry of the theory. For example, the four-dimensional $\mathcal{N}=4$ theory has the $S O(6)$ $R$ symmetry, which we identify as the rotational group in the transverse directions to the brane. We classify the operators according to the $R$ symmetry since operators with different $R$ charges are renormalized differently and carry different conformal weights. The situation changes in noncommutative theories discussed in this paper. We note that the open Wilson line (3) does not belong to a definite representation of the $R$ symmetry, but rather it is parametrized by the vector $y_{\alpha}$ in the transverse directions. By 
expanding in powers of $y^{\alpha}$, one finds that the Wilson line is a superposition of infinitely many representations of the $R$ symmetry. We show that it in fact matches well with an asymptotic behavior of a solution to a wave equation in the background geometry for the dual gravitational description. This observation also helps us clarify some aspects of the correspondence between operators in the gauge theory and boundary conditions in the dual gravitational description.

This paper is organized as follows. In Section 2, we compute the disk amplitudes and derive the coupling of the noncommutative gauge fields to closed string states in the bulk. After a description of our set-up, we start with a computation of a bosonic string $S$-matrix involving a closed string tachyon and arbitrary number of gauge fields. By taking the zero slope limit, we find that the tachyon in the bulk couples to the straight open Wilson line (3) satisfying the constraint (5). We then study the coupling to the graviton in the bulk and derive the energy-momentum tensor on the brane, in both bosonic string theory and superstring theory. The structure of gauge theory operators coupled to massive string modes is briefly described. In Section 3, we discuss the dual gravitational descriptions of the noncommutative theories. A detailed derivation of the energy-momentum tensor and a proof of its conservation in the case of bosonic string are presented in Appendices A and B.

After completion of the work, we were informed that Thomas Mehen and Mark Wise have independently computed a disk amplitude of one graviton and two gauge fields in bosonic string theory. Their work overlaps with some of the results presented in this paper.

\section{Disk Amplitudes and Open Wilson Lines}

In this section, we study the disk amplitude. The worldsheet disk is represented by the upper half-plane of the complex plane, and the propagator is given by [33 - 35]

$$
\begin{aligned}
\left\langle X^{M}(z) X^{N}(w)\right\rangle=-\alpha^{\prime} & {\left[g^{M N} \log |z-w|-g^{M N} \log |z-\bar{w}|+\right.} \\
+ & \left.2 G^{M N} \log |z-\bar{w}|+\frac{1}{2 \pi \alpha^{\prime}} \theta^{M N} \log \left(\frac{z-\bar{w}}{\bar{z}-w}\right)\right] .
\end{aligned}
$$

We decompose the coordinates $X^{M}$ into three directions: $M=(\alpha, \mu, i)$ where $\alpha$ runs in the directions transverse to the brane, $\mu$ and $i$ in the commutative and noncommutative directions, respectively, along the branes. Therefore, $\theta^{M N}=0$ unless $(M, N)=(i, j)$. The closed string metric is denoted by $g_{M N}$, so the open string metric $G_{M N}$ on the brane $M=\mu, i$ is given by

$$
G^{\mu \nu}=g^{\mu \nu}, \quad G^{i j}=\frac{1}{\left(2 \pi \alpha^{\prime}\right)^{2}} \theta^{i m} \theta^{j n} g_{n m}
$$


in the zero slope limit [10]. We also set

$$
G^{\alpha \beta}=0,
$$

so that the propagator (6) obeys the correct Dirichlet boundary conditions in the transverse directions. The noncommutative gauge theory is defined by taking the zero slope limit $\alpha^{\prime} \rightarrow 0$, while keeping $G^{i j}$ and $\theta^{i j}$ finite.

The amplitudes we consider contain one closed string with momentum $k$ and an arbitrary number of open strings with momenta $p_{a}(a=1, \ldots, n)$. In string theory, we can only compute on-shell physical amplitudes. In the zero slope limit, the gauge field $A_{M}$ on the brane can have any amount of momentum since the on-shell condition,

$$
\alpha^{\prime} G^{M N} p_{M} p_{N}=0
$$

holds for any finite $p$. Another way to say this is that the open string operator $e^{i p X}$ does not receive anomalous dimensions when $\alpha^{\prime}=0$.

Once we choose the open string momenta, the closed string momentum $k$ along the brane is determined by the momentum conservation $k+p_{1}+\cdots+p_{n}=0$. Since the inverse $g^{i j}$ of the closed string metric scales as $\alpha^{\prime-2}$ in the noncommutative direction, if $k_{i}$ is non-zero and finite, $g^{i j} k_{i} k_{j}$ is large $\sim \alpha^{\prime-2}$ and positive. Therefore, to maintain the closed string on-shell condition

$$
\alpha^{\prime} g^{M N} k_{M} k_{N}=4(1-\mathbf{N}) \quad(\mathbf{N}: \text { amount of closed string excitations })
$$

the closed string has to have an energy which grows as $\alpha^{\prime-1}$. This is a well-known fact and reflects the decoupling of closed string modes from the gauge theory degrees of freedom on the brane [36 - 38]. In order to keep the momentum $k=-\left(p_{1}+\cdots+p_{n}\right)$ along the brane to be finite, we assume that the brane is oriented in spacelike directions so that the $O\left(\alpha^{\prime-1}\right)$ component of $k$ is transverse to the brane. ${ }^{2}$ The scaling $k_{\alpha} \sim \alpha^{\prime-1}$ fits well with the fact that the scalar fields $\Phi^{\alpha}$ describing the location of the branes in the transverse directions couple to $y_{\alpha}=2 \pi \alpha^{\prime} k_{\alpha}$.

\footnotetext{
${ }^{2}$ Alternatively we may take the brane to be oriented in space-time directions by allowing the transverse momentum to be pure imaginary. This set-up is more appropriate when we apply our results to the dual gravitational description of large $N$ theories $[31,32]$, which we will discuss in Section 3. In the dual description, the gauge theory operators are located at the boundary of the bulk geometry. They are in fixed momentum states in the noncommutative directions but can be localized in the commutative directions. The insertion of a gauge theory operator is realized by imposing a boundary condition on the corresponding bulk field that it grows exponentially toward the boundary points where the operators are inserted but decays exponentially toward elsewhere. Namely, the bulk field carries an imaginary momentum in the transverse directions. Everything we say in the following is unchanged in this set-up, except that we need to make the substitution $y_{\alpha} \rightarrow i y_{\alpha}$ in some of the equations. We may also consider the case when the bulk metric is Euclidean, again by allowing the transverse momentum to be imaginary.
} 
In the limit $\alpha^{\prime} \rightarrow 0$, the on-shell condition (10) reduces to

$$
G_{i j} \theta^{i m} \theta^{j n} k_{m} k_{n}+g^{\alpha \beta} y_{\alpha} y_{\beta}=0 .
$$

We note that this condition is independent of the amount $\mathbf{N}$ of closed string excitations. We can then study the coupling of arbitrary excitations of closed string to the gauge theory on the brane while keeping open string momenta to be finite and arbitrary.

It is also important to note that the momentum $k_{\mu}$ in the commutative directions on the brane does not appear in the on-shell condition (11). Thus one can perform the inverse Fourier transformation on $k_{\mu}$ to obtain gauge theory operators localized in the commutative directions. On the other hand, it is not possible to perform the inverse Fourier transform on $k_{i}$ in the noncommutative directions if one tries to keep $y_{\alpha}$ fixed since $k_{i}$ and $y_{\alpha}$ are related by (11).

\subsection{Tachyon in Bosonic String}

Let us start with the one-point function of the close string tachyon $V(z)=e^{i k X(z)}$ with arbitrary number of open strings in bosonic string theory. As a warm-up exercise, we first examine the contraction of the closed string vertex $V(z)$ with the $e^{i p X(t)}$ part of the open string vertex, where $z$ is in the upper half plane and $t$ is on the real axis. In the zero slope limit, the bulk-boundary propagator in the worldsheet is simplified as

$$
\left\langle X^{M}(z) X^{N}(t)\right\rangle=-i \theta^{M N} \tau(t, z)+O\left(\alpha^{\prime}\right),
$$

where the function $\tau(t, z)$ is given by

$$
\tau(t, z)=\frac{1}{2 \pi i} \log \left(\frac{t-z}{t-\bar{z}}\right) .
$$

For a fixed value of $z, \tau(t, z)$ is a monotonically increasing function of $t$ and

$$
\tau(\infty, z)-\tau(-\infty, z)=1
$$

We choose the branch of the logarithm in (13) so that $\tau(-\infty, z)=0$. The amplitude in the zero slope limit is then

$$
\begin{aligned}
& (z-\bar{z})^{2}\left\langle e^{i k X(z)} e^{i p_{1} X\left(t_{1}\right)} \cdots e^{i p_{n} X\left(t_{n}\right)}\right\rangle \\
= & \exp \left[-\frac{i}{2} \sum_{a<b} p_{a} \theta p_{b} \epsilon\left(t_{a}-t_{b}\right)+\sum_{a} i k \theta p_{a} \tau\left(t_{a}, z\right)\right] \delta\left(k+p_{1}+\cdots+p_{n}\right) .
\end{aligned}
$$

Here $\epsilon(t)$ is the step function which is equal to 1 or -1 for positive and negative $t$. The factor $(z-\bar{z})^{2}$ on the left-hand side of the equation cancels a factor due to selfcontraction of the closed string vertex operator $e^{i k X}$. We used the on-shell condition for the tachyon,

$$
\alpha^{\prime} g^{M N} k_{M} k_{N}=4
$$


but the open string momenta $p_{a}$ are arbitrary. In fact, the right-hand side of (15) is $S L(2, R)$ invariant for any $p_{a}$ since

$$
\begin{aligned}
& \sum_{a} k \theta p_{a} \tau\left(\frac{\alpha t_{a}+\beta}{\gamma t_{a}+\delta}, \frac{\alpha z+\beta}{\gamma z+\delta}\right) \\
= & \sum_{a} k \theta p_{a} \tau\left(t_{a}, z\right)+k \theta\left(\sum_{a} p_{a}\right) \frac{1}{2 \pi i} \log \left(\frac{\gamma \bar{z}+\delta}{\gamma z+\delta}\right) \\
= & \sum_{a} k \theta p_{a} \tau\left(t_{a}, z\right)
\end{aligned}
$$

where

$$
\alpha \delta-\beta \gamma=1, \quad \alpha, \beta, \gamma, \delta: \text { real, }
$$

and we used $k \theta \sum_{a} p_{a}=-k_{i} \theta^{i j} k_{j}=0$. Since the open string momenta $p_{a}$ are arbitrary in the zero slope limit, we can take the inverse Fourier transform of (15) with respect to $p_{a}$ with the total momentum fixed and obtain,

$$
(z-\bar{z})^{2}\left\langle e^{i k X(z)} \prod_{a} f_{a}\left(X\left(t_{a}\right)\right)\right\rangle=\int d x *\left[e^{i k x} \prod_{a} f_{a}\left(x+l \tau\left(t_{a}, z\right)\right)\right] .
$$

Here $l^{i}=k_{j} \theta^{j i}$ and the integral $\int d x$ is over the brane. The symbol $*[\cdots]$ means that we evaluate the expression in [...] using the standard star product with the ordering along the boundary of the worldsheet as,

$$
\begin{aligned}
& *\left[f_{1}\left(x+\tau\left(t_{1}, z\right)\right) f_{2}\left(x+\tau\left(t_{2}, z\right)\right)\right] \\
= & \left.\exp \left(\frac{i}{2} \epsilon\left(t_{1}-t_{2}\right) \theta^{i j} \frac{\partial^{2}}{\partial \xi^{i} \partial \zeta^{j}}\right) f_{1}\left(x+l \tau\left(t_{1}, z\right)+\xi\right) f_{2}\left(x+l \tau\left(t_{2}, z\right)+\zeta\right)\right|_{\xi, \zeta=0} .
\end{aligned}
$$

We note that the locations of the open strings are displaced from the closed string location $x^{i}$ by the amount $l^{i} \tau\left(t_{a}, z\right)$, which runs from 0 to $l^{i}$ as $t_{a}$ goes from $-\infty$ to $\infty$.

Now we are ready to consider the vertex operator for gauge field,

$$
U(t)=\left(u_{i}(p) \frac{d X^{i}(t)}{d t}+u_{\mu}(p) \frac{d X^{\mu}(t)}{d t}\right) e^{i p X(t)} .
$$

Here $i$ and $\mu$ run in the noncommutative and commutative directions, respectively, along the brane. For the polarization $u_{i}$ in the noncommutative directions, we can contract $d X^{i}(t) / d t$ with $e^{i k X(z)}$ using (12). The zero slope limit of such an amplitude is

$$
(z-\bar{z})^{2}\left\langle e^{i k X(z)} \prod_{a=1}^{n} e^{i p_{a} X\left(t_{a}\right)} u_{i}\left(p_{a}\right) \frac{d X^{i}}{d t_{a}}\right\rangle
$$




$$
\begin{aligned}
=\exp [ & \left.-\frac{i}{2} \sum_{a<b} p_{a} \theta p_{b} \epsilon\left(t_{a}-t_{b}\right)+\sum_{a} i k \theta p_{a} \tau\left(t_{a}, z\right)\right] \times \\
& \times \prod_{a} l^{i} u_{i}\left(p_{a}\right) \frac{\partial \tau\left(t_{a}, z\right)}{\partial t_{a}} \delta\left(k+p_{1}+\cdots+p_{n}\right) .
\end{aligned}
$$

In the coordinate basis, this takes the form,

$$
\begin{aligned}
& (z-\bar{z})^{2}\left\langle e^{i k X(z)} \prod_{a} A_{i}\left(X\left(t_{a}\right)\right) \frac{d X^{i}}{d t_{a}}\right\rangle \\
= & \int d x *\left[e^{i k x} \prod_{a} l^{i} A_{i}\left(x+l \tau\left(t_{a}, z\right)\right) \frac{\partial \tau\left(t_{a}, z\right)}{\partial t_{a}}\right] .
\end{aligned}
$$

We can exponentiate this result to write ${ }^{3}$

$$
\begin{aligned}
& (z-\bar{z})^{2}\left\langle e^{i k X(z)} \exp \left(i \int_{-\infty}^{\infty} d t A_{i}(X(t)) \frac{d X^{i}}{d t}\right)\right\rangle \\
= & \int d x *\left[e^{i k x} \exp \left(i \int_{0}^{1} d \tau l^{i} A_{i}(x+l \tau)\right)\right] .
\end{aligned}
$$

On the right-hand side of the equation, we changed integration variables from $t$ to $\tau=\tau(t, z)$. Thus we find that the disk amplitude with a closed string naturally leads to the straight open Wilson line connecting $x^{i}$ and $x^{i}+l^{i}$. Following the definition of the star product in (20), the path-ordering in (24) is such that $A_{i}(x)$ is to the left. It is clear that, when there are several branes, the computation including the ChanPaton factor gives the contraction of the gauge group indices of $A_{i}$ according to this path-ordering.

On the other hand, the polarization $u_{\mu}$ in the commutative direction costs $\alpha^{\prime}$ every time we contract $d X^{\mu} / d t$ with the closed string vertex $e^{i k X}$. Thus terms involving $u_{\mu}$ are subleading in the $\alpha^{\prime}$ expansion.

We can also consider the vertex operator for the scalar fields

$$
U(t)=\phi^{\alpha}(p) i g_{\alpha \beta} \partial_{\perp} X^{\beta} e^{i p X}
$$

where $\partial_{\perp}$ is the derivative in the direction transverse to the boundary of the worldsheet. As in the case of the polarization in the commutative direction along the brane, the contraction of $i g_{\alpha \beta} \partial_{\perp} X^{\beta}$ with the closed string vertex $e^{i k X} \operatorname{costs} \alpha^{\prime}$. However this is compensated by the fact that the closed string momentum $k_{\alpha}$ in the transverse direction scales as $\alpha^{\prime-1}$. Thus disk amplitudes with the scalar fields $\Phi^{\alpha}$ are of the same order in

\footnotetext{
${ }^{3}$ In this paper we use the point-splitting regularization on the worldsheet, which is known to lead to the standard description of the noncommutative gauge theory [10].
} 
$\alpha^{\prime}$ as those with the gauge field $A_{i}$ in the noncommutative directions. By computation similar to the one that led to (24), using

$$
i \partial_{\perp} \log \left|z-t_{1}\right|^{2}=\frac{z-\bar{z}}{\left(z-t_{1}\right)\left(\bar{z}-t_{1}\right)}
$$

we find

$$
\begin{aligned}
& (z-\bar{z})^{2}\left\langle e^{i k X(z)} \exp \left(i \int_{-\infty}^{\infty} d t \Phi^{\alpha}(X(t)) i g_{\alpha \beta} \partial_{\perp} X^{\beta}\right)\right\rangle \\
= & \int d x *\left[e^{i k x} \exp \left(i \int_{0}^{1} d \tau y_{\alpha} \Phi^{\alpha}(x+l \tau)\right)\right],
\end{aligned}
$$

where

$$
y_{\alpha}=2 \pi \alpha^{\prime} k_{\alpha}
$$

and $\Phi^{\alpha}(x)$ is the Fourier transform of $\phi^{\alpha}(p)$. Again the path-ordered exponential is implicit in this expression.

We can combine the amplitudes for the gauge field $\left(A_{\mu}, A_{i}\right)$ and the scalar field $\Phi^{\alpha}$ to write

$$
\begin{aligned}
& (z-\bar{z})^{2}\left\langle e^{i k X(z)} \exp \left(i \int_{-\infty}^{\infty} d t A_{i}(X) \frac{d X^{i}}{d t}+A_{\mu}(X) \frac{d X^{\mu}}{d t}+\Phi^{\alpha}(X) i g_{\alpha \beta} \partial_{\perp} X^{\beta}\right)\right\rangle \\
= & \int d x *\left[e^{i k x} \exp \left(i \int_{0}^{1} d \tau l^{i} A_{i}(x+l \tau)+y_{\alpha} \Phi^{\alpha}(x+l \tau)\right)\right]
\end{aligned}
$$

It is interesting to note that, because of the on-shell condition (16), $l^{i}=k_{j} \theta^{j i}$ and $y_{\alpha}$ in (29) are related as

$$
G_{i j} l^{i} l^{j}+g^{\alpha \beta} y_{\alpha} y_{\beta}=0 .
$$

It is straightforward to turn (29) into the string theory $S$-matrix computation and to read off the coupling between the closed string tachyon and the noncommutative gauge field. Let us look at the tachyon- $A_{i}$ amplitude (23). Because of the $S L(2, R)$ invariance, we can fix the locations $z$ of the closed string vertex and of one of the open string vertices, say $t_{1}$. The ghost factor is

$$
\left\langle c(z) \bar{c}(\bar{z}) c\left(t_{1}\right)\right\rangle=(z-\bar{z})\left(z-t_{1}\right)\left(\bar{z}-t_{1}\right) .
$$

Since

$$
\frac{\partial \tau\left(t_{1}, z\right)}{\partial t_{1}}=\frac{1}{2 \pi i} \frac{z-\bar{z}}{\left(z-t_{1}\right)\left(\bar{z}-t_{1}\right)}
$$

we can combine (23) and (31) to write

$$
\begin{aligned}
& 2 \pi i\left\langle c(z) \bar{c}(\bar{z}) c\left(t_{1}\right)\right\rangle\left\langle e^{i k X(z)} \prod_{a} A_{i}\left(X\left(t_{a}\right)\right) \frac{d X^{i}}{d t_{a}}\right\rangle \\
= & \int d x *\left[e^{i k x} l^{i} A_{i}\left(x+l \tau\left(t_{1}, z\right)\right) \prod_{a=2}^{n} l^{j} A_{j}\left(x+l \tau\left(t_{a}, z\right)\right) \frac{\partial \tau\left(t_{a}, z\right)}{\partial t_{a}}\right] .
\end{aligned}
$$


The $S$-matrix is obtained by integrating $t_{2}, \ldots, t_{n}$ along the real axis, taking into account the ordering of the open string vertex operators. By the $S L(2, R)$ invariance, the result of the integral is independent of $z$ and $t_{1}$,

$$
\begin{aligned}
& \int_{-\infty}^{\infty} d t_{2} \cdots d t_{n} 2 \pi i\left\langle c(z) \bar{c}(\bar{z}) c\left(t_{1}\right)\right\rangle\left\langle e^{i k X(z)} \prod_{a} A_{i}\left(X\left(t_{a}\right)\right) \frac{d X^{i}}{d t_{a}}\right\rangle \\
= & \int d x *\left[e^{i k x} \prod_{a=1}^{n} \int_{0}^{1} d \tau_{a} l^{j} A_{j}\left(x+l \tau_{a}\right)\right] .
\end{aligned}
$$

We can then exponentiate the result as in (24). The computation involving the scalar field $\Phi^{\alpha}$ is essentially the same except that we need to use (26) in place of (32).

The last step is to remove one-particle reducible (1PR) parts of gauge fields from the $S$-matrix. In fact, the $1 \mathrm{PR}$ parts have already been removed in our computation at the zero slope limit. This is more or less obvious from the fact that there is no operator product singularities (33) at $t_{a}=t_{b}$. To see this more explicitly, we need to take $\alpha^{\prime}$ to be small but non-zero. A massless pole in the $S$-matrix arises from an integral of the form,

$$
\int d t_{a} \frac{\alpha^{\prime}}{t_{a}-t_{b}}\left(t_{a}-t_{b}\right)^{2 \alpha^{\prime} p_{a} \cdot p_{b}} \sim \frac{1}{p_{a} \cdot p_{b}},
$$

where $p_{a} \cdot p_{b}=G^{i j} p_{a i} p_{b j}+G^{\mu \nu} p_{a \mu} p_{b \nu}$. The factor $\left(t_{a}-t_{b}\right)^{2 \alpha^{\prime} p_{a} \cdot p_{b}}$ is due to the contraction of $e^{i p_{a} X}$ and $e^{i p_{b} X}$ at finite $\alpha^{\prime}$. The additional factor $\alpha^{\prime} /\left(t_{a}-t_{b}\right)$ comes from a contraction of $d X / d t$ in one of the open string vertex operators with another. This additional singularity $\left(t_{a}-t_{b}\right)^{-1}$ is needed in order to get the massless pole. Because of the form of the propagator (6), the singularity $\left(t_{a}-t_{b}\right)^{-1}$ is always accompanied by a factor of $\alpha^{\prime}$. In our computation, we have taken the limit $\alpha^{\prime} \rightarrow 0$ before we do any integral over $t_{a}$ 's. If we take the limit in this order, the left-hand side of (35) vanishes. Thus there is no $1 \mathrm{PR}$ parts of gauge fields in the $S$-matrix we computed in (34).

To summarize, we have shown that the closed string tachyon couples to the open Wilson line defined by

$$
\mathcal{O}_{\text {tachyon }}=\int d x *\left[e^{i\left(k_{\mu} x^{\mu}+k_{i} x^{i}\right)} \exp \left(i \int_{0}^{1} d \tau l^{i} A_{i}(x+l \tau)+y_{\alpha} \Phi^{\alpha}(x+l \tau)\right)\right]
$$

This operator is gauge invariant provided $l^{i}=k_{j} \theta^{j i}[14]$. The vectors $l^{i}$ and $y_{\alpha}$ have to obey the constraint (30) in order for the operator to couple to the tachyon.

\subsection{Graviton in Bosonic String}

The vertex operators of massless closed string modes are of the form,

$$
V(z)=h_{M N}(k) \partial X^{M} \bar{\partial} X^{N} e^{i k X(z)} .
$$


We will consider the case when $h_{M N}$ is a symmetric tensor, corresponding to the graviton and the dilaton in the bulk. We are particularly interested in the graviton polarized in the noncommutative directions since the corresponding gauge theory operator can be regarded as the energy-momentum tensor $T^{i j}$ of the gauge theory.

The Wick contraction of $\langle V(z) \times$ open strings $\rangle$ generates various terms. Let us first examine the terms that are generated by using the first two terms in the propagator (6), i.e.,

$$
\left\langle X^{M}(z) X^{N}(w)\right\rangle_{\text {the first two }}=-\alpha^{\prime} g^{M N} \log \left|\frac{z-w}{z-\bar{w}}\right| .
$$

This vanishes when either $z$ or $w$ is on the boundary, so we can use this only for the self-contraction of the closed string vertex operator $\partial X^{M} \bar{\partial} X^{N} e^{i k X(z)}$. After removing a divergent factor by the normal ordering of the operator, the resulting term is proportional to $g^{M N}$ and we can identify it as coming from the dilaton part of $V(z)$. (The contraction of $\partial X^{i}$ or $\bar{\partial} X^{j}$ with $e^{i k X}$ using (38) generates other terms. However they vanish because of the physical state condition $h_{M N} g^{N N^{\prime}} k_{N^{\prime}}=0$.)

Now let us turn to the graviton part of $V(z)$. We are interested in the case when the graviton is polarized in the noncommutative directions. As we did in the previous subsection, let us start with a warm-up exercise by paying attention to the $e^{i p X}$ part of the open string vertex operators. Since the first two terms in (6) contribute only to the dilaton part of $V(z)$ and the third term is of the order $O\left(\alpha^{\prime}\right)$, we can compute the contraction of $V(z)$ with $e^{i p X}$ using only the fourth term in the propagator (6),

$$
\left\langle X^{i}(z) X^{j}(w)\right\rangle_{\text {the fourth }}=-\frac{1}{2 \pi} \theta^{i j} \log \left(\frac{z-\bar{w}}{\bar{z}-w}\right) .
$$

The result is

$$
\begin{gathered}
(z-\bar{z})^{2}\left\langle\partial X^{i} \bar{\partial} X^{j} e^{i k X(z)} \prod_{a=1}^{n} e^{i p_{a} X\left(t_{a}\right)}\right\rangle \\
=(z-\bar{z})^{2} \exp \left[-\frac{i}{2} \sum_{a<b} p_{a} \theta p_{b} \epsilon\left(t_{a}-t_{b}\right)+\sum_{a} i k \theta p_{a} \tau\left(t_{a}, z\right)\right] \delta\left(k+p_{1}+\cdots+p_{n}\right) \times \\
\quad \times \sum_{a, b=1}^{n} \frac{1}{2 \pi i}\left(\frac{(\theta k)^{i}}{z-\bar{z}}+\sum_{a=1}^{n} \frac{\left(\theta p_{a}\right)^{i}}{z-t_{a}}\right) \frac{1}{2 \pi i}\left(\frac{(\theta k)^{j}}{z-\bar{z}}-\sum_{b=1}^{n} \frac{\left(\theta p_{b}\right)^{j}}{\bar{z}-t_{b}}\right) \\
=\exp \left[-\frac{i}{2} \sum_{a<b} p_{a} \theta p_{b} \epsilon\left(t_{a}-t_{b}\right)+\sum_{a} i k \theta p_{a} \tau\left(t_{a}, z\right)\right] \delta\left(k+p_{1}+\cdots+p_{n}\right) \times \\
\times \frac{1}{(2 \pi i)^{2}} \sum_{a, b=1}^{n} e^{-2 \pi i \tau\left(t_{a}, z\right)+2 \pi i \tau\left(t_{b}, z\right)}\left(\theta p_{a}\right)^{i}\left(\theta p_{b}\right)^{j}
\end{gathered}
$$

To go from the second to the third line, we used the momentum conservation $k=$ 
$-\left(p_{1}+\cdots+p_{n}\right)$ and the identities,

$$
\frac{1}{z-\bar{z}}-\frac{1}{z-t}=\frac{1}{z-\bar{z}} e^{-2 \pi i \tau(z, t)}, \quad \frac{1}{z-\bar{z}}+\frac{1}{\bar{z}-t}=\frac{1}{z-\bar{z}} e^{+2 \pi i \tau(z, t)} .
$$

The inverse Fourier transform of this then gives

$$
\begin{aligned}
& (z-\bar{z})^{2}\left\langle\partial X^{i} \bar{\partial} X^{j} e^{i k X(z)} \prod_{a=1}^{n} f_{a}\left(X\left(t_{a}\right)\right)\right\rangle \\
= & \frac{1}{(2 \pi)^{2}} \int d x *\left[e^{i k x} \sum_{a, b=1}^{n} e^{-2 \pi i \tau\left(t_{a}, z\right)+2 \pi i \tau\left(t_{b}, z\right)} \theta^{i m} \theta^{j n} \frac{\partial^{2}}{\partial x_{a}^{m} \partial x_{b}^{n}} \prod_{c=1}^{n} f_{c}\left(x_{c}\right)\right],
\end{aligned}
$$

where we set

$$
x_{a}^{i}=x^{i}+l^{i} \tau\left(t_{a}, z\right), \quad a=1, \ldots, n,
$$

after taking the derivatives on the right-hand side of (42). We see that the one-point function of the graviton vertex operator is given by acting the second order differential operator,

$$
\sum_{a, b} e^{-2 \pi i \tau\left(t_{a}, z\right)+2 \pi i \tau\left(t_{b}, z\right)} \theta^{i m} \theta^{j n} \frac{\partial^{2}}{\partial x_{a}^{m} \partial x_{b}^{m}},
$$

on the corresponding formula (19) for the one-point function of the close string tachyon.

We are now ready to discuss the amplitudes involving the gauge fields $A_{i}, A_{\mu}$ and the scalar fields $\Phi^{\alpha}$. As one can expect, we need to replace the partial derivatives in (44) with the covariant derivatives as in

$$
\begin{aligned}
& F_{i j}=\partial_{i} A_{j}-\partial_{j} A_{i}+i\left(A_{i} * A_{j}-A_{j} * A_{i}\right), \\
& D_{i} \Phi^{\alpha}=\partial_{i} \Phi^{\alpha}+i\left(A_{i} * \Phi^{\alpha}-\Phi^{\alpha} * A_{i}\right) .
\end{aligned}
$$

The nonlinear terms in the covariant derivatives arise from integration by parts in the integrals over the locations $t_{a}$ of the open string vertex operators, taking into account the fact that we are using the point splitting regularization on the worldsheet. As in the case of the closed string tachyon state discussed in the previous subsection, it is straightforward to turn this into the string theory $S$-matrix computation and to read off the coupling of the graviton to the gauge fields and the scalar fields on the branes. We find that the bulk graviton $h_{i j}$ polarized in the noncommutative directions couples to the gauge theory operator $T^{i j}$ given by

$$
\begin{gathered}
T^{i j}=\frac{\theta^{i i^{\prime}} \theta^{j j^{\prime}}+\theta^{j i^{\prime}} \theta^{i j^{\prime}}}{(2 \pi)^{2}} \int d x *\left[e^{i k x} \exp \left(i \int_{0}^{1} d \tau l^{i} A_{i}(x+l \tau)+y_{\alpha} \Phi^{\alpha}(x+l \tau)\right) \times\right. \\
\times\left\{i \int_{0}^{1} d \tau e^{-2 \pi i \tau}\left(l^{m} F_{i^{\prime} m}(x+l \tau)+y_{\alpha} D_{i^{\prime}} \Phi^{\alpha}(x+l \tau)\right) \times\right. \\
\quad \times i \int_{0}^{1} d \tau^{\prime} e^{+2 \pi i \tau^{\prime}}\left(l^{n} F_{j^{\prime} n}\left(x+l \tau^{\prime}\right)+y_{\beta} D_{j^{\prime}} \Phi^{\beta}\left(x+l \tau^{\prime}\right)\right)+ \\
\left.\left.\quad+i \int_{0}^{1} d \tau\left(l^{m} D_{i^{\prime}} F_{j^{\prime} m}(x+l \tau)+y_{\alpha} D_{i^{\prime}} D_{j^{\prime}} \Phi^{\alpha}(x+l \tau)\right)\right\}\right]
\end{gathered}
$$


A detailed derivation of this formula is presented in Appendix A.

There are several interesting aspects of this operator $T^{i j}$. It has been expected in the literature that the operators in the noncommutative gauge theory dual to particle states in the bulk take the form,

$$
\widehat{\mathcal{O}}(k)=\int d x *\left[e^{i k x} \operatorname{STr} \prod_{i} \int_{0}^{1} d \tau B_{i}(x+l \tau) \exp \left(i \int_{0}^{1} d \tau l^{i} A_{i}(x+l \tau)\right)\right],
$$

when the corresponding operators in the theory in commutative space are given by

$$
\mathcal{O}(k)=\int d x e^{i k x} \operatorname{STr} \prod_{i} B_{i}(x) .
$$

(Here we have written the symmetrized trace STr over the gauge group index explicitly.) The expression (46) we found for $T^{i j}$ does not follow this prescription. One of the motivation for the conjecture (47) was that it is the simplest solution to the requirement that $\widehat{\mathcal{O}}(k)$ reduces to $\mathcal{O}(k)$ in the commutative limit,

$$
\widehat{\mathcal{O}}(k) \rightarrow \mathcal{O}(k), \quad \text { as } \quad \theta^{i j} \rightarrow 0 .
$$

The operator $T^{i j}$ we have derived does not satisfy this requirement. Instead it vanishes in this limit because of the factor $\left(\theta^{i i^{\prime}} \theta^{j j^{\prime}}+\theta^{i j^{\prime}} \theta^{j i^{\prime}}\right)$ in front of everything.

This result may seem paradoxical since the worldsheet propagator (6) we have used to derive (46) reduces to the one for the commutative case if we substitute $\theta^{i j}=0$ and $g^{i j}=G^{i j}$. To clarify the situation, it is useful to point out that the amplitude (42) and the corresponding formula for the gauge fields computed in Appendix A do not contain any factor of $\alpha^{\prime}$. These amplitudes in the noncommutative case are non-zero and finite in the zero slope limit. It is because they are computed using the propagator (39), which does not contain $\alpha^{\prime}$. This is to be contrasted to the commutative case when the standard expression for the energy-momentum tensor

$$
T_{\text {commutative }}^{i j} \sim F^{i}{ }_{k} F^{k j}+F^{i}{ }_{\mu} F^{\mu j}-\frac{1}{4} G^{i j} F^{2}+\cdots,
$$

is obtained from $O\left(\alpha^{\prime 3}\right)$ terms in the disk amplitude of one graviton and several gauge fields. The amplitude that leads to (50) involves at least 3 propagators on the worldsheet, each of which costs one power of $\alpha^{\prime}$. The fact that $T^{i j}$ in the noncommutative case vanishes in the limit $\theta^{i j} \rightarrow 0$ is consistent with the fact that there is no $O(1)$ terms in the commutative case. This resolves the apparent paradox.

Another interesting aspect of the energy-momentum tensor $T^{M N}$ is the way it is conserved,

$$
k_{M} T^{M N}=0 .
$$


Let us first examine the conservation of $T^{M j}$, which involves $T^{i j}, T^{\mu j}$ and $T^{\alpha j}$. One can show that the leading terms in the mixed components $T^{\mu j}$ and $T^{\alpha j}$ are of the order $O\left(\alpha^{\prime}\right)$. Since $k_{i}, k_{\alpha} \sim O(1)$ and $k_{\alpha} \sim O\left(\alpha^{\prime-1}\right)$, the conservation law at the zero slope limit is

$$
k_{i} T^{i j}+k_{\alpha} T^{\alpha j}=k_{i} T^{i j}+\frac{y_{\alpha}}{2 \pi \alpha^{\prime}} T^{\alpha j}=0 .
$$

The expression for $T^{\alpha j}$ is

$$
\begin{aligned}
& T^{\alpha j}=\alpha^{\prime} \theta^{j m} \int d x *\left[e^{i k x} \exp \left(i \int_{0}^{1} d \tau l^{i} A_{i}(x+l \tau)+y_{\alpha} \Phi^{\alpha}(x+l \tau)\right) \times\right. \\
& \times\left\{\int_{0}^{1} d \tau e^{-2 \pi i \tau} \Phi^{\alpha}(x+l \tau) \int_{0}^{1} d \tau^{\prime} e^{+2 \pi i \tau^{\prime}} i\left(l^{n} F_{m n}\left(x+l \tau^{\prime}\right)+y_{\beta} D_{m} \Phi^{\beta}\left(x+l \tau^{\prime}\right)\right)\right. \\
& \left.\left.-\int_{0}^{1} d \tau e^{+2 \pi i \tau} \Phi^{\alpha}(x+l \tau) \int_{0}^{1} d \tau^{\prime} e^{-2 \pi i \tau^{\prime}} i\left(l^{n} F_{m n}\left(x+l \tau^{\prime}\right)+y_{\beta} D_{m} \Phi^{\beta}\left(x+l \tau^{\prime}\right)\right)\right\}\right] .
\end{aligned}
$$

Curiously, we do not have to use the equation of motion of the gauge fields and the scalar fields in order to prove the conservation law (52). It just follows from the kinematical identities such as

$$
\begin{aligned}
& k_{i} \theta^{i i^{\prime}} l^{m} F_{i^{\prime} m}=l^{i} l^{j} F_{i j}=0, \\
& k_{i} \theta^{i i^{\prime}} \int_{0}^{1} d \tau \partial_{i^{\prime}} f(x+l \tau)=\int_{0}^{1} d \tau \partial_{\tau} f(x+l \tau)=f(x+l)-f(x) .
\end{aligned}
$$

The first line in (54) vanished because of the antisymmetry of $F_{i j}$. The right-hand side of the second line vanishes when it is inserted in $\int d x *\left[e^{i k x} \cdots\right]$, taking into account the path-ordering along the Wilson line. A detailed proof of the conservation law (52) is given in Appendix B. To describe salient features of the proof, let us look at $e^{-2 \pi i \tau} \theta^{i i^{\prime}}\left(l^{m} F_{i^{\prime} m}+y_{\alpha} D_{i^{\prime}} \Phi^{\alpha}\right)$ in the second line of (46). If we multiply $k_{i}$ to it, the first term vanishes because of the first identity in (54). As for the second term, we can use the second identity in (54) to perform the integration by parts. Because of the factor $e^{-2 \pi i \tau}$, we obtain an additional term of the form $2 \pi i \Phi^{\alpha}$ from the integration by parts. It is canceled by $y_{\alpha} T^{\alpha j}$ because of the second line in (53) multiplied by $y_{\alpha}$.

Similarly one can prove the conservation of $T^{M \beta}$,

$$
k_{i} T^{i \beta}+k_{\alpha} T^{\alpha \beta}=k_{i} T^{i \alpha}+\frac{y_{\alpha}}{2 \pi \alpha^{\prime}} T^{\alpha \beta}=0
$$

where $T^{\alpha \beta}$ is

$$
\begin{aligned}
& T^{\alpha \beta}=-\left(2 \pi \alpha^{\prime}\right)^{2} \int d x *\left[e^{i k x} \exp \left(i \int_{0}^{1} d \tau l^{i} A_{i}(x+l \tau)+y_{\alpha} \Phi^{\alpha}(x+l \tau)\right) \times\right. \\
& \left.\quad \times\left\{\int_{0}^{1} d \tau e^{-2 \pi i \tau} \Phi^{\alpha}(x+l \tau) \int_{0}^{1} d \tau^{\prime} e^{+2 \pi i \tau^{\prime}} \Phi^{\beta}\left(x+l \tau^{\prime}\right)+(\alpha \leftrightarrow \beta)\right\}\right] .
\end{aligned}
$$


Let us discuss implications of the fact that the energy-momentum tensor is kinematically conserved. It is well-known that, given a symmetry of a Lagrangian, the construction of the corresponding Nöther current is not unique since one can add a term of the form $\partial_{j} J^{i j}$ where $J^{i j}$ is anti-symmetric in $i$ and $j$. Such a term is conserved without using any equation of motion. This ambiguity has a physical origin; when the current is coupled to a gauge field $a_{i}$, the term $\partial_{j} J^{i j}$ represents the coupling to the field strength,

$$
\left(\partial_{i} a_{j}-\partial_{j} a_{i}\right) J^{i j}
$$

One can say that $\partial_{j} J^{i j}$ is conserved without using the equation of motion since the term (57) does not modify the equation of motion when the gauge field is flat. A similar term can arise in the energy-momentum tensor when the matter couples directly to the curvature of the metric. Such a coupling does not modify the equation of motion in flat space but changes the form of the energy-momentum tensor. An example of this is found in the Coulomb-Gas formalism of two-dimensional conformal field theory where one modifies the energy-momentum tensor of the massless free scalar field $\varphi$ as

$$
\begin{array}{lll}
T_{z z}=\frac{1}{2} \partial \varphi^{2} & \rightarrow & \frac{1}{2} \partial \varphi^{2}+\kappa \partial^{2} \varphi \\
T_{z \bar{z}}=0 & \rightarrow & -\kappa \partial \bar{\partial} \varphi
\end{array}
$$

with some constant $\kappa$. These additional terms have physical significance. For example, the conformal anomaly of the theory depends on $\kappa$.

The fact that the energy-momentum tensor $\left(T^{i j}, T^{\alpha j}\right)$ presented in (46) and (53) is conserved without using the equation of motion suggests that there is a coupling of the bulk metric to the noncommutative gauge theory which vanishes in the limit of flat space. It is curious that such a coupling appears in the leading order in the $\alpha^{\prime}$ expansion. It would be interesting to compute amplitudes involving more gravitons and to identify the precise form of the coupling.

\subsection{Graviton in Superstring}

To compute the disk amplitude of one graviton and many gauge fields in superstring theory, it is convenient to use the operator in the $(-1,-1)$-picture for the graviton,

$$
V^{(-1,-1)}(z)=\frac{1}{2} \delta(\gamma) \delta(\bar{\gamma}) h_{M N}(k) \psi^{M}(z) \bar{\psi}^{N}(\bar{z}) e^{i k X(z)}
$$

and the operator in the 0-picture for open string,

$$
\begin{aligned}
U^{(0)}(t)=u_{i}(p)\left(\frac{d X^{i}}{d t}\right. & \left.-2 i p \cdot \Psi \Psi^{i}\right) e^{i p X}+u_{\mu}(p)\left(\frac{d X^{\mu}}{d t}-2 i p \cdot \Psi \Psi^{\mu}\right) e^{i p X}+ \\
& +\phi^{\alpha}(p) g_{\alpha \beta}\left(i \partial_{\perp} X^{\beta}-2 i p \cdot \Psi \Psi^{\beta}\right) e^{i p X}
\end{aligned}
$$


where $\gamma$ and $\bar{\gamma}$ are bosonic ghosts, $\psi$ and $\bar{\psi}$ are worldsheet fermions in the left and the right movers, and $p \cdot \Psi=p_{i} \Psi^{i}+p_{\mu} \Psi^{\mu}$. In the noncommutative directions, the fermion propagators are given by

$$
\begin{aligned}
\left\langle\psi^{i}(z) \psi^{j}(w)\right\rangle & =\frac{\alpha^{\prime}}{z-w} g^{i j}, \\
\left\langle\psi^{i}(z) \bar{\psi}^{j}(\bar{w})\right\rangle & =\frac{\alpha^{\prime}}{z-\bar{w}}\left(-g^{i j}+2 G^{i j}+2 \frac{\theta^{i j}}{2 \pi \alpha^{\prime}}\right), \\
\left\langle\bar{\psi}^{i}(\bar{z}) \psi^{j}(w)\right\rangle & =\frac{\alpha^{\prime}}{\bar{z}-w}\left(-g^{i j}+2 G^{i j}-2 \frac{\theta^{i j}}{2 \pi \alpha^{\prime}}\right), \\
\left\langle\bar{\psi}^{i}(\bar{z}) \bar{\psi}^{j}(\bar{w})\right\rangle & =\frac{\alpha^{\prime}}{\bar{z}-\bar{w}} g^{i j},
\end{aligned}
$$

and the fermions on the boundary are defined by

$$
\begin{aligned}
\Psi^{i} & =\frac{1}{2}\left(\psi^{i}+\bar{\psi}^{i}\right), \quad \Psi^{\mu}=\frac{1}{2}\left(\psi^{\mu}+\bar{\psi}^{\mu}\right), \\
\Psi^{\alpha} & =\frac{1}{2}\left(\psi^{\alpha}-\bar{\psi}^{\alpha}\right) .
\end{aligned}
$$

The bulk-boundary propagators are

$$
\begin{aligned}
\left\langle\psi^{i}(z) \Psi^{j}(t)\right\rangle & =\frac{\alpha^{\prime}}{z-t}\left(G^{i j}+\frac{\theta^{i j}}{2 \pi \alpha^{\prime}}\right) \\
\left\langle\bar{\psi}^{i}(\bar{z}) \Psi^{j}(t)\right\rangle & =\frac{\alpha^{\prime}}{\bar{z}-t}\left(G^{i j}-\frac{\theta^{i j}}{2 \pi \alpha^{\prime}}\right) .
\end{aligned}
$$

Using these, we can show in the leading order in the $\alpha^{\prime}$ expansion that

$$
\begin{gathered}
(z-\bar{z})\left\langle\frac{1}{2}\left(\psi^{i} \bar{\psi}^{j}+\psi^{j} \bar{\psi}^{i}\right) e^{i k X(z)} \exp \left(i \int_{-\infty}^{\infty} d t A_{i} \frac{d X^{i}}{d t}-F_{i j} \Psi^{i} \Psi^{j}\right)\right\rangle \\
=2 \theta^{i i^{\prime}} \theta^{j j^{\prime}} \int d x *\left[e^{i k x} \exp \left(i \int_{0}^{1} d \tau l^{i} A_{i}(x+l \tau)\right) \times\right. \\
\left.\times \alpha^{\prime} G^{m n} \int_{0}^{1} d \tau\left(F_{i^{\prime} m}(x+l \tau)-\theta_{i^{\prime} m}^{-1}\right) \int_{0}^{1} d \tau^{\prime}\left(F_{j^{\prime} n}\left(x+l \tau^{\prime}\right)-\theta_{j^{\prime} n}^{-1}\right)\right] .
\end{gathered}
$$

As usual we have removed contributions from the dilaton part of the closed string vertex operator. The nonlinear terms in the field strength $F_{i j}$ on the right-hand side are generated as a consequence of the point-splitting regularization on the left-hand side [10]. Similar expressions can be derived for amplitudes involving $A_{\mu}$ and $\Phi^{\alpha}$.

As we did in the previous two subsections, we can convert (64) into the superstring $S$-matrix computation using,

$$
\langle\delta(\gamma(z)) \delta(\bar{\gamma}(\bar{z}))\rangle=\frac{1}{z-\bar{z}}
$$


and

$$
\left\langle c(z) \bar{c}(\bar{z}) c\left(t_{1}\right)\right\rangle=(z-\bar{z})\left(z-t_{1}\right)\left(\bar{z}-t_{1}\right),
$$

and extract the gauge theory operator which couples to the bulk graviton. The result is

$$
\begin{aligned}
& T^{i j}=2 \alpha^{\prime} \theta^{i i^{\prime}} \theta^{j j^{\prime}} \int d x *\left[e^{i k x} \exp \left(i \int_{0}^{1} d \tau l^{i} A_{i}(x+l \tau)+y_{\alpha} \Phi^{\alpha}(x+l \tau)\right) \times\right. \\
& \times \int_{0}^{1} d \tau \int_{0}^{1} d \tau^{\prime}\left(G^{m n}\left(F_{i^{\prime} m}(x+l \tau)-\theta_{i^{\prime} m}^{-1}\right)\left(F_{j^{\prime} n}\left(x+l \tau^{\prime}\right)-\theta_{j^{\prime} n}^{-1}\right)+\right. \\
& \left.\left.\quad+G^{\mu \nu} F_{i^{\prime} \mu}(x+l \tau) F_{j^{\prime} \nu}\left(x+l \tau^{\prime}\right)+g_{\alpha \beta} D_{i^{\prime}} \Phi^{\alpha}(x+l \tau) D_{j^{\prime}} \Phi^{\beta}\left(x+l \tau^{\prime}\right)\right)\right] .
\end{aligned}
$$

The way the energy-momentum tensor is conserved is again very interesting. Consider $k_{i} T^{i j}$ and pay attention to the second line of (67). The multiplication of $k_{i}$ generates a term of the form $\int_{0}^{1} d \tau l^{i} F_{i m}(z+l \tau)$, and this can be written as $D_{m}$ acting on the $A_{i}$ part of the Wilson line, $\exp \left(i \int_{0}^{1} d \tau l^{i} A_{i}(x+l \tau)\right)$. We can then perform integration by parts in $D_{m}$. This gives rise to two terms. One is of the form $k_{m} G^{m n} F_{j^{\prime} n}$, which is generated by acting $D_{m}$ on $e^{i k x}$. It is cancelled by the terms in the second line of (67) containing $\theta^{-1}$. The other is of the form $D_{m} G^{m n} F_{j^{\prime} n}$ and makes a part of the equation of motion for the gauge fields. A more careful analysis shows that $k_{M} T^{M j}$ vanishes because of the equation of motion.

The fact that the conservation of the energy-momentum requires the equation of motion for the gauge fields means that, unlike the case of bosonic string, the coupling of the bulk metric to the gauge fields does not disappear in the limit of flat space. In fact the expression for the energy-momentum tensor (67) is consistent with the way the bulk metric appears in action for the noncommutative gauge theory derived in [10],

$$
\begin{aligned}
S=\frac{-1}{g_{Y M}^{2}} \int d x & \sqrt{\operatorname{det} G} *\left[\frac{1}{4} G^{M N} G^{P Q}\left(F_{M P}-\theta_{M P}^{-1}\right)\left(F_{N Q}-\theta_{N Q}^{-1}\right)+\right. \\
& \left.+\frac{1}{2} G^{M N} g_{\alpha \beta} D_{M} \Phi^{\alpha} D_{N} \Phi^{\beta}-\frac{1}{4} g_{\alpha \beta} g_{\gamma \delta}\left[\Phi^{\alpha}, \Phi^{\gamma}\right]\left[\Phi^{\beta}, \Phi^{\delta}\right]\right] .
\end{aligned}
$$

Here $\theta_{M N}^{-1}=0$ unless $(M, N)=(i, j)$. The action depends on the closed string metric $g_{i j}$ through the open string metric

$$
G^{i j}=\frac{1}{\left(2 \pi \alpha^{\prime}\right)^{2}} \theta^{i m} \theta^{j m} g_{n m},
$$

and the gauge coupling constant

$$
g_{Y M}^{2}=\frac{2 \pi g_{\text {string }}}{\sqrt{\operatorname{det}\left(\frac{\theta^{i m} g_{m j}}{2 \pi \alpha^{\prime}}\right)}}
$$


where $g_{\text {string }}$ is the string coupling constant. A variation of the action with respect to $g_{i j}$ gives

$$
\begin{gathered}
\frac{\partial S}{\partial g_{i j}}=\frac{-\theta^{i i^{\prime}} \theta^{j j^{\prime}}}{2\left(2 \pi \alpha^{\prime}\right)^{2} g_{Y M}^{2}} \int d x \sqrt{\operatorname{det} G} *\left[G^{m n}\left(F_{i^{\prime} m}-\theta_{i^{\prime} m}^{-1}\right)\left(F_{j^{\prime} n}-\theta_{j^{\prime} n}^{-1}\right)+\right. \\
\left.+G^{\mu \nu} F_{i^{\prime} \mu} F_{j^{\prime} \nu}+g_{\alpha \beta} D_{i^{\prime}} \Phi^{\alpha} D_{j^{\prime}} \Phi^{\beta}\right] .
\end{gathered}
$$

To derive this, we used the fact that the combination $\sqrt{\operatorname{det} G} / g_{Y M}^{2}$ is independent of the closed string metric $g_{i j}$. Since the action (68) was derived assuming the bulk metric is flat, we can only make the variation with respect to constant $g_{i j}$. It is satisfactory to see that our result (67) agrees with (71) in the limit $k \rightarrow 0$.

It is important to point out that the expression for the energy-momentum tensor $(67)$ still does not reduce to the one in the commutative case (50) in the limit $\theta^{i j} \rightarrow 0$. In the string theory computation, (67) comes from the order $\alpha^{\prime}$ terms of the disk amplitude whereas the commutative result is from the order $\alpha^{\prime 3}$. The difference in the powers of $\alpha^{\prime}$ in these computations is manifest in the factor $\alpha^{\prime-2}$ on the right-hand side of (71).

\subsection{Massive String Modes}

Disk amplitudes for massive modes of close string can be evaluated in the same way as we did for the tachyon and the massless modes. The basic ingredient is the open Wilson line connecting $x$ and $x+l$ with the condition

$$
G_{i j} l^{i} l^{j}+g^{\alpha \beta} y_{\alpha} y_{\beta}=0
$$

As we discussed earlier, this condition is independent of the amount $\mathbf{N}$ of closed string excitations. Each $\partial X^{i}$ in the closed string vertex operator $V(z)$ is involved in the selfcontraction within $V(z)$ (as in the case of the dilaton) or introduces the gauge theory operator $e^{-2 \pi i \tau} \theta^{i j}\left(l^{m} F_{j m}(x+l \tau)+\cdots\right)$ integrated along the Wilson line (as in the case of the graviton). More derivatives on $\partial X^{i}$ induce more derivatives on $F_{j m}$, etc.

\section{Comments on Gravity Dual}

In this paper, we studied coupling of noncommutative gauge theories on branes to close string states in the bulk. We showed that a basic ingredient is the straight open Wilson line. It may seem paradoxical that a closed string can couple to something that is open. This is possible since the distance between the two end-point of the Wilson

line measured by the closed string metric, $\sqrt{g_{i j} l^{i} l^{j}}$, is of the order $\alpha^{\prime}$, which is much smaller than the string length $\sqrt{\alpha^{\prime}}$. Therefore, closed string states of the size of $\sqrt{\alpha^{\prime}}$ in the bulk can easily couple to the open Wilson line of the length $\sim \alpha^{\prime}$. 
There are several large $N$ noncommutative gauge theories that are expected to have dual gravitational descriptions, generalizing the $A d S / C F T$ correspondence [39] to the case when the NS-NS two-form field is non-trivial [31, 32, 40]. For the four-dimensional $\mathcal{N}=4$ gauge theory with two noncommutative directions, the metric of the dual gravitational theory is $[31,32]$

$$
\begin{gathered}
\frac{d s^{2}}{\sqrt{\lambda} \alpha^{\prime}}=\frac{d u^{2}}{u^{2}}+u^{2} G_{\mu \nu} d x^{\mu} d x^{\nu}+\frac{u^{2}}{1+\lambda \theta^{2} u^{4}} G_{i j} d x^{i} d x^{j}+d \Omega_{5}^{2}, \\
\lambda=g_{Y M}^{2} N,
\end{gathered}
$$

where the metric is given in the string frame, $\mu, \nu=0,1$ and $i, j=2,3$ with $\theta=\theta^{23}$, and $d \Omega_{5}^{2}$ is the metric on the unit 5 -sphere. Near the boundary $u \rightarrow \infty$, the metric in the commutative directions $\left(x_{0}, x_{1}\right)$ behaves as in $A d S$. Relative to this, the metric in the noncommutative directions $\left(x_{2}, x_{3}\right)$ scales as $u^{-4}$. This scaling is equivalent to $g_{i j} \sim \alpha^{\prime 2}$ used throughout this paper if we identify $u$ as the UV cutoff scale of the gauge theory. Therefore, for the same reason as in the previous paragraph, particles states in the bulk can couple to the open Wilson line located at the boundary of the geometry (73). It is not necessary to look for a classical string worldsheet ending on the Wilson line at the boundary; such a description breaks down for large $u$ when the size of the Wilson line measured by the bulk metric becomes smaller than the string length.

There has been evidence that correlation functions of supergravity fields computed in the geometry (73) agree with gauge theory expectations [13, 41]. To make a quantitative comparison between the gauge theory and its gravity dual, we need to know a precise map between gauge theory operators and supergravity fields. We believe the present paper provides a framework to answer this question.

The gauge theory operators we constructed are in fixed momentum states in the noncommutative directions but can be localized in the commutative directions along the brane. This is because the momentum $k_{\mu}$ in the commutative directions is not constrained by the on-shell condition (11). Moreover, the operators are parametrized by $y_{\alpha}$, which is a vector in the transverse directions. In the case of the four-dimensional $\mathcal{N}=4$ theory, $y_{\alpha}$ is a 6 -dimensional vector obeying the on-shell condition ${ }^{4}$

$$
g^{\alpha \beta} y_{\alpha} y_{\beta}=G_{i j} \theta^{i m} \theta^{j n} k_{m} k_{n}
$$

For a given $k_{i}$, we may regard $y_{\alpha}$ as coordinates on $S^{5}$. By expanding the Wilson line in powers of $y_{\alpha}$, we generate infinitely many representations of the $S O(6) R$ symmetry. This is to be contrasted to the case of the theory on commutative space. There operators are classified into irreducible representations of the $R$ symmetry since operators with different $R$ charges are renormalized differently and have different conformal weights.

\footnotetext{
${ }^{4}$ Here we made the replacement $y_{\alpha} \rightarrow i y_{\alpha}$ as we discussed in the footnote 2 in Section 2.
} 
We would like to point out that the construction of Wilson lines in the noncommutative theory fits well with its dual gravitational description. To see how it works, it would be useful to remind ourselves of the situation of the commutative case. There the gravity dual is the string theory on $A d S_{5} \times S^{5}$ with the metric,

$$
\frac{d s^{2}}{\sqrt{g_{Y M}^{2} N} \alpha^{\prime}}=\frac{d u^{2}}{u^{2}}+u^{2}\left(-d x_{0}^{2}+d x_{1}^{2}+d x_{2}^{2}+d x_{3}^{2}\right)+d \Omega_{5}^{2} .
$$

Consider the Klein-Gordon equation in this geometry for a scalar field with mass $m$. For an eigenstate of the momentum in the $x$ direction and the angular momentum in the $S^{5}$ direction, the equation becomes

$$
\left(-u^{2} \frac{d^{2}}{d u^{2}}+\tilde{m}^{2}+j(j+4)+\frac{15}{4}+\frac{k^{2}}{u^{2}}\right) u^{5 / 2} \Psi(u)=0,
$$

where $j$ is the orbital angular momentum of $S O(6)$, which we regard as the $R$ charge, and $\tilde{m}^{2}=m^{2} \sqrt{g_{Y M}^{2} N} \alpha^{\prime}$. For large $u$, a solution to this behaves as

$$
\Psi(u) \sim u^{\Delta_{ \pm}-4}\left(1+O\left(\frac{1}{u}\right)\right), \quad u \rightarrow \infty
$$

where

$$
\Delta_{ \pm}=2 \pm \sqrt{\tilde{m}^{2}+(j+2)^{2}}
$$

It is important to note that the exponent of $u$ depends on the $R$ charge $j$ as well as the mass $\tilde{m}$. Thus operators with different $j$ scale differently as $u \rightarrow \infty$. On the other hand, since the momentum $k$ does not appear in the scaling behavior in (77), we can take the inverse Fourier transformation with respect to $k$ and consider a source localized at a point $x_{0}$ on the boundary. Namely, we can impose a boundary condition on the scalar field $\Psi$ so that it behaves near the boundary as

$$
\Psi \sim u^{\Delta_{+}-4} \delta^{(4)}\left(x-x_{0}\right), \quad u \rightarrow \infty .
$$

The exponent of $u$ is then related to the conformal weight of the corresponding operator in the gauge theory inserted at $x_{0}$. This gives the correspondence between operators in the gauge theory and boundary conditions on the bulk fields $[39,42,43]$.

Now let us turn to the geometry (73) for the noncommutative gauge theory. In this background, the dilaton $\phi$ also depends on $u$,

$$
e^{2 \phi}=\frac{\lambda}{N} \frac{1}{1+\lambda \theta^{2} u^{4}} .
$$

For definiteness, we consider a massive scalar field minimally coupled to the metric in the Einstein frame, $g_{M N}^{E}=e^{-\phi / 2} g_{M N}$. The equation corresponding to (76) is then

$$
\left(-u^{2} \frac{d^{2}}{d u^{2}}+u^{2} \lambda G_{i j} l^{i} l^{j}+\tilde{m}^{2}\left(1+\lambda \theta^{2} u^{4}\right)^{\frac{1}{4}}+\right.
$$




$$
\left.+j(j+4)+\frac{15}{4}+\frac{G^{\mu \nu} k_{\mu} k_{\nu}+G^{i j} k_{i} k_{j}}{u^{2}}\right) u^{5 / 2} \Psi(u)=0
$$

where $l^{i}=k_{j} \theta^{j i}$ as usual and $\tilde{m}$ is the mass with some rescaling. For large $u$, a solution to this equation is

$$
\Psi(u)=u^{\lambda_{ \pm}} e^{ \pm u \sqrt{\lambda G_{i j} l^{i} l^{j}}}\left(1+O\left(\frac{1}{u}\right)\right)
$$

where

$$
\lambda_{ \pm}=-\frac{5}{2} \pm \frac{\tilde{m} \theta^{1 / 2}}{2 \sqrt{\sqrt{\lambda} G_{i j} l^{i} l^{j}}} .
$$

The $u$-dependent factor on the right-hand side are to be removed by the renormalization of the corresponding operator. ${ }^{5}$ We note that these terms are independent of the $R$ charge $j$ as well as the momentum $k_{\mu}$ in the commutative directions along the brane. Therefore, we can perform the inverse Fourier transformation on them and construct a solution which grows exponentially toward a point $\Omega_{0}$ on $S^{5}$ and $x_{0}$ in the commutative directions on the boundary. We have found that the wave equation in the geometry (73) allows us to impose the boundary condition

$$
\Psi \sim u^{\lambda_{+}} e^{u \sqrt{\lambda G_{i j} l^{i} l^{j}}} \delta^{(2)}\left(x-x_{0}\right) \delta^{(5)}\left(\Omega-\Omega_{0}\right), \quad u \rightarrow \infty .
$$

It is natural to interpret it as a boundary condition corresponding to an insertion of a gauge theory operator at the point $x_{0}$ in the commutative direction. The point $\Omega_{0}$ on $S^{5}$ specifies the parameter $y_{\alpha}$ in the Wilson line. Given the boundary condition (84), one can solve the wave equation in the bulk, compute a coefficient in front of the exponentially decaying part $\sim e^{-u \sqrt{\lambda G_{i j} l^{i} l^{j}}}$ of the solution and extract information on the strongly coupled dynamics of the noncommutative gauge theory as discussed in $[31,32,13]$.

We have found that, in the noncommutative case, it is possible to impose a boundary condition on a particle state in the bulk that it ends on a point on $S^{5}$ as well as on a point in the commutative directions. This is to be contrasted to the commutative case when point particle states in $A d S_{5} \times S^{5}$ are classified into irreducible representations of $S O(6)$. The boundary condition in the noncommutative case reminds us of the one for Wilson loop in the gravitational dual of the $\mathcal{N}=4$ theory in commutative space $[44,45]$. There a loop is parametrized by $x^{\mu}(t)(\mu=0, \ldots, 3)$ and $y_{\alpha}(t)(\alpha=1, \ldots, 6)$ obeying the condition [46],

$$
G_{\mu \nu} \dot{x}^{\mu} \dot{x}^{\nu}+g^{\alpha \beta} \dot{y}_{\alpha} \dot{y}_{\beta}=0
$$

\footnotetext{
${ }^{5}$ The linear term in the exponent $\pm \sqrt{\lambda G_{i j} l^{i} l^{j}} u$ means that $\Psi(u)$ has an imaginary momentum in the $u$ direction. This fits well with our discussion at the beginning of Section 2 and in the footnote 2 .
} 
similar to the on-shell condition (5) in our case. The loop variables $(x(t), y(t))$ specify a boundary condition on a semi-classical string worldsheet on $A d S_{5} \times S^{5}$ that it terminates at a loop $x(t)$ on the boundary of $A d S_{5}$ and $\dot{y}(t)$ on $S^{5}$. In this respect, a particle state in the noncommutative case behaves similarly to a semi-classical worldsheet in the commutative case.

In this paper, we developed a method to determine gauge theory operators dual to any closed string states including massive ones. In particular, we found the precise form of the gauge theory operator $T^{i j}$ coupled to the bulk graviton polarized in the noncommutative directions in both bosonic string theory and superstring theory. In either case, the expression is different from what was expected in the literature. In the case of bosonic string, the structure of $T^{i j}$ suggests that the leading coupling between the gauge fields on the brane and the graviton in the bulk is through the curvature of the bulk metric. In the case of superstring, the expression for $T^{i j}$ is consistent with the way the bulk metric appears in the action of the noncommutative gauge theory [10].

\section{Acknowledgments}

We would like to thank Jaume Gomis, Thomas Mehen, John Schwarz, Mark Wise and

Edward Witten for discussions. The research was supported in part by the DOE grant DE-FG03-92ER40701 and the Caltech Discovery Fund. 


\section{A Energy-Momentum Tensor $T^{i j}$}

In this appendix, we give a detailed derivation of the operator $T^{i j}$ in the case of bosonic string theory. Let us first consider vertex operators for $A_{i}$,

$$
(z-\bar{z})^{2}\left\langle\partial X^{i} \bar{\partial} X^{j} e^{i k X(z)} \prod_{a=1}^{n} u_{l}\left(p_{a}\right) \frac{d X^{l}}{d t_{a}} e^{i p_{a} X\left(t_{a}\right)}\right\rangle .
$$

There are four types of contractions;

1. Both $\partial X^{i}$ and $\bar{\partial} X^{j}$ contract with $e^{i k X(z)}$ or $e^{i p X(t)}$.

The computation is essentially the same as (40) and the result is given by

$$
\begin{aligned}
& \exp \left[-\frac{i}{2} \sum_{a<b} p_{a} \theta p_{b} \epsilon\left(t_{a}-t_{b}\right)+\sum_{a} i k \theta p_{a} \tau\left(t_{a}, z\right)\right] \delta\left(k+p_{1}+\cdots+p_{n}\right) \times \\
& \times \sum_{a, b=1}^{n} \frac{i\left(\theta p_{a}\right)^{i}}{2 \pi} e^{-2 \pi i \tau\left(t_{a}, z\right)} \frac{i\left(\theta p_{b}\right)^{j}}{2 \pi} e^{2 \pi i \tau\left(t_{b}, z\right)} \prod_{c=1}^{n} k \theta u_{c} \frac{\partial \tau\left(t_{c}, z\right)}{\partial t_{c}}
\end{aligned}
$$

where $u_{c}$ is an abbreviation for $u\left(p_{c}\right)$ and we use the same notation in what follows. 2. Both $\partial X^{i}$ and $\bar{\partial} X^{j}$ contract with $d X^{l} / d t$.

$$
\begin{aligned}
& \exp \left[-\frac{i}{2} \sum_{a<b} p_{a} \theta p_{b} \epsilon\left(t_{a}-t_{b}\right)+\sum_{a} i k \theta p_{a} \tau\left(t_{a}, z\right)\right] \delta\left(k+p_{1}+\cdots+p_{n}\right) \times \\
& \times \sum_{a \neq b}-i\left(\theta u_{a}\right)^{i} e^{-2 \pi i \tau\left(t_{a}, z\right)} \frac{\partial \tau\left(t_{a}, z\right)}{\partial t_{a}} i\left(\theta u_{b}\right)^{j} e^{2 \pi i \tau\left(t_{b}, z\right)} \frac{\partial \tau\left(t_{b}, z\right)}{\partial t_{b}} \times \\
& \times \prod_{c \neq a, b} k \theta u_{c} \frac{\partial \tau\left(t_{c}, z\right)}{\partial t_{c}}
\end{aligned}
$$

where we used

$$
\frac{1}{(z-t)^{2}}=\frac{2 \pi i}{z-\bar{z}} e^{-2 \pi i \tau(t, z)} \frac{\partial \tau(t, z)}{\partial t}, \quad \frac{1}{(\bar{z}-t)^{2}}=\frac{2 \pi i}{z-\bar{z}} e^{2 \pi i \tau(t, z)} \frac{\partial \tau(t, z)}{\partial t} .
$$

3. $\partial X^{i}$ contracts with $d X^{l} / d t$ and $\bar{\partial} X^{j}$ contracts with $e^{i k X(z)}$ or $e^{i p X(t)}$.

$$
\begin{aligned}
& \exp \left[-\frac{i}{2} \sum_{a<b} p_{a} \theta p_{b} \epsilon\left(t_{a}-t_{b}\right)+\sum_{a} i k \theta p_{a} \tau\left(t_{a}, z\right)\right] \delta\left(k+p_{1}+\cdots+p_{n}\right) \times \\
& \times \sum_{a, b=1}^{n}-i\left(\theta u_{a}\right)^{i} e^{-2 \pi i \tau\left(t_{a}, z\right)} \frac{\partial \tau\left(t_{a}, z\right)}{\partial t_{a}} \frac{i\left(\theta p_{b}\right)^{j}}{2 \pi} e^{2 \pi i \tau\left(t_{b}, z\right)} \prod_{c \neq a} k \theta u_{c} \frac{\partial \tau\left(t_{c}, z\right)}{\partial t_{c}} .
\end{aligned}
$$

4. $\partial X^{i}$ contracts with $e^{i k X(z)}$ or $e^{i p X(t)}$ and $\bar{\partial} X^{j}$ contracts with $d X^{l} / d t$.

$$
\begin{aligned}
& \exp \left[-\frac{i}{2} \sum_{a<b} p_{a} \theta p_{b} \epsilon\left(t_{a}-t_{b}\right)+\sum_{a} i k \theta p_{a} \tau\left(t_{a}, z\right)\right] \delta\left(k+p_{1}+\cdots+p_{n}\right) \times \\
& \times \sum_{a, b=1}^{n} \frac{i\left(\theta p_{a}\right)^{i}}{2 \pi} e^{-2 \pi i \tau\left(t_{a}, z\right)} i\left(\theta u_{b}\right)^{j} e^{2 \pi i \tau\left(t_{b}, z\right)} \frac{\partial \tau\left(t_{b}, z\right)}{\partial t_{b}} \prod_{c \neq b} k \theta u_{c} \frac{\partial \tau\left(t_{c}, z\right)}{\partial t_{c}} .
\end{aligned}
$$


These four contractions are combined to give

$$
\begin{aligned}
& \exp \left[-\frac{i}{2} \sum_{a<b} p_{a} \theta p_{b} \epsilon\left(t_{a}-t_{b}\right)\right] \delta\left(k+p_{1}+\cdots+p_{n}\right) \times \\
& \times\left[\sum_{a \neq b}\left(\frac{i\left(\theta p_{a}\right)^{i} k \theta u_{a}}{2 \pi}-i\left(\theta u_{a}\right)^{i}\right) e^{i\left(k \theta p_{a}-2 \pi\right) \tau\left(t_{a}, z\right)} \frac{\partial \tau\left(t_{a}, z\right)}{\partial t_{a}} \times\right. \\
& \times\left(\frac{i\left(\theta p_{b}\right)^{j} k \theta u_{b}}{2 \pi}+i\left(\theta u_{b}\right)^{j}\right) e^{i\left(k \theta p_{b}+2 \pi\right) \tau\left(t_{b}, z\right)} \frac{\partial \tau\left(t_{b}, z\right)}{\partial t_{b}} \prod_{c \neq a, b} k \theta u_{c} e^{i k \theta p_{c} \tau\left(t_{c}, z\right)} \frac{\partial \tau\left(t_{c}, z\right)}{\partial t_{c}}+ \\
& \left.+\sum_{a=1}^{n} \frac{i\left(\theta p_{a}\right)^{i}}{2 \pi} \frac{i\left(\theta p_{a}\right)^{j}}{2 \pi} k \theta u_{a} e^{i k \theta p_{a} \tau\left(t_{a}, z\right)} \frac{\partial \tau\left(t_{a}, z\right)}{\partial t_{a}} \prod_{c \neq a} k \theta u_{c} e^{i k \theta p_{c} \tau\left(t_{c}, z\right)} \frac{\partial \tau\left(t_{c}, z\right)}{\partial t_{c}}\right] .
\end{aligned}
$$

The factor $i\left(\theta p_{a}\right)^{i} k \theta u_{a}$ corresponds to $\theta^{i m} \partial_{m} A_{n} l^{n}$ in the coordinate basis. We will show how this becomes the covariant form $\theta^{i m} F_{m n} l^{n}$. Note that

$$
\frac{i(\theta p)^{i} k \theta u}{2 \pi} \mp i(\theta u)^{i}=\frac{i(\theta p)^{i} k \theta u-i(\theta u)^{i}(k \theta p)}{2 \pi}+\frac{(\theta u)^{i}}{2 \pi} i(k \theta p \mp 2 \pi),
$$

where the first term on the right-hand side corresponds to $\theta^{i m}\left(\partial_{m} A_{n}-\partial_{n} A_{m}\right) l^{n}$ divided by $2 \pi$ in the coordinate basis and the second term is a total derivative:

$$
\frac{(\theta u)^{i}}{2 \pi} i(k \theta p \mp 2 \pi) e^{i(k \theta p \mp 2 \pi) \tau}=\frac{(\theta u)^{i}}{2 \pi} \frac{\partial}{\partial \tau} e^{i(k \theta p \mp 2 \pi) \tau} .
$$

Now consider surface terms in the path-ordered exponential when we integrate with respect to $\tau_{n}$. We have

$$
\begin{aligned}
& \quad \int_{\tau_{n-2}}^{1} d \tau_{n-1} i l^{n} A_{n}\left(x+l \tau_{n-1}\right) \int_{\tau_{n-1}}^{1} d \tau_{n} \frac{\partial}{\partial \tau_{n}}\left(\frac{i \theta^{i m}}{2 \pi} A_{m}\left(x+l \tau_{n}\right) e^{\mp 2 \pi i \tau_{n}}\right) \times \\
& \quad \times \int_{\tau_{n}}^{1} d \tau_{n+1} i l^{n} A_{n}\left(x+l \tau_{n+1}\right) \int_{\tau_{n+1}}^{1} d \tau_{n+2} \ldots \\
& =-\int_{\tau_{n-2}}^{1} d \tau_{n-1} \frac{i^{2} \theta^{i m}}{2 \pi} l^{n} A_{n}\left(x+l \tau_{n-1}\right) A_{m}\left(x+l \tau_{n-1}\right) e^{\mp 2 \pi i \tau_{n-1}} \times \\
& \quad \times \int_{\tau_{n-1}}^{1} d \tau_{n+1} i l^{n} A_{n}\left(x+l \tau_{n+1}\right) \int_{\tau_{n+1}}^{1} d \tau_{n+2} \ldots \\
& +\int_{\tau_{n-2}}^{1} d \tau_{n-1} i l^{n} A_{n}\left(x+l \tau_{n-1}\right) \times \\
& \times \int_{\tau_{n-1}}^{1} d \tau_{n} \frac{i^{2} \theta^{i m}}{2 \pi} A_{m}\left(x+l \tau_{n}\right) l^{n} A_{n}\left(x+l \tau_{n}\right) e^{\mp 2 \pi i \tau_{n}} \int_{\tau_{n}}^{1} d \tau_{n+2} \ldots,
\end{aligned}
$$

where the $*$ product is implicit. The surface contributions precisely provide the nonlinear terms $i A_{m} * A_{n}-i A_{n} * A_{m}$ in $F_{m n}$. Thus we have shown that the first part in (92) can be written in the coordinate basis as follows:

$$
\frac{i \theta^{i m}}{2 \pi} \frac{i \theta^{j n}}{2 \pi} \int_{0}^{1} d \tau^{\prime} e^{-2 \pi i \tau^{\prime}} l^{m^{\prime}} F_{m m^{\prime}}\left(x+l \tau^{\prime}\right) \int_{0}^{1} d \tau^{\prime \prime} e^{2 \pi i \tau^{\prime \prime}} l^{n^{\prime}} F_{n n^{\prime}}\left(x+l \tau^{\prime \prime}\right) .
$$


The second part in (92) contains the factor which corresponds to $\theta^{i m} \theta^{j n} l^{n^{\prime}} \partial_{m} \partial_{n} A_{n^{\prime}}$ in the coordinate basis. We expect that this factor turns into the gauge covariant form $\theta^{i m} \theta^{j n} l^{n^{\prime}} D_{m} F_{n n^{\prime}}$ by a manipulation similar to the one described in the above and by taking into account extra surface contributions which arise when the two integrals with respect to $\tau^{\prime}$ and $\tau^{\prime \prime}$ in (96) are next to each other in the path-ordered exponential. The result for $A_{i}$ is

$$
\begin{aligned}
& (z-\bar{z})^{2}\left\langle\partial X^{i} \bar{\partial} X^{j} e^{i k X(z)} \exp \left(i \int_{-\infty}^{\infty} d t A_{i}(X) \frac{d X^{i}}{d t}\right)\right\rangle \\
= & \int d x *\left[e^{i k x} \exp \left(i \int_{0}^{1} d \tau l^{i} A_{i}(x+l \tau)\right) \times\right. \\
& \times\left\{\frac{i \theta^{i m}}{2 \pi} \frac{i \theta^{j n}}{2 \pi} \int_{0}^{1} d \tau^{\prime} e^{-2 \pi i \tau^{\prime}} l^{m^{\prime}} F_{m m^{\prime}}\left(x+l \tau^{\prime}\right) \int_{0}^{1} d \tau^{\prime \prime} e^{2 \pi i \tau^{\prime \prime}} l^{n^{\prime}} F_{n n^{\prime}}\left(x+l \tau^{\prime \prime}\right)+\right. \\
+ & \left.\left.\frac{i \theta^{i m} \theta^{j n}}{(2 \pi)^{2}} \int_{0}^{1} d \tau^{\prime} l^{n^{\prime}} D_{m} F_{n n^{\prime}}\left(x+l \tau^{\prime}\right)\right\}\right]
\end{aligned}
$$

The generalization to the case involving other fields $A_{\mu}$ and $\Phi^{\alpha}$ is rather straightforward. It is obvious that terms involving $A_{\mu}$ are subleading in $\alpha^{\prime}$ just as in the case of the coupling to the closed string tachyon. On the other hand, the vertex operator $\partial_{\perp} X^{\alpha}$ can produce an $O(1)$ contribution if it is contracted with the $e^{i k X(z)}$ part of the closed string vertex operator. Therefore, only the first type of the contractions described at the beginning of this appendix is allowed for $\partial_{\perp} X^{\alpha}$ and the effect of including the scalar field is the following replacements in (92)

$$
\begin{aligned}
\frac{i\left(\theta p_{a}\right)^{i} k \theta u_{a}}{2 \pi} \mp i\left(\theta u_{a}\right)^{i} & \rightarrow \frac{i\left(\theta p_{a}\right)^{i} k \theta u_{a}}{2 \pi} \mp i\left(\theta u_{a}\right)^{i}+\frac{i\left(\theta p_{a^{\prime}}\right)^{i} y_{\alpha} \phi^{\alpha}\left(p_{a^{\prime}}\right)}{2 \pi}, \\
\frac{i\left(\theta p_{a}\right)^{i}}{2 \pi} \frac{i\left(\theta p_{a}\right)^{j}}{2 \pi} k \theta u_{a} & \rightarrow \frac{i\left(\theta p_{a}\right)^{i}}{2 \pi} \frac{i\left(\theta p_{a}\right)^{j}}{2 \pi} k \theta u_{a}+\frac{i\left(\theta p_{a^{\prime}}\right)^{i}}{2 \pi} \frac{i\left(\theta p_{a^{\prime}}\right)^{j}}{2 \pi} y_{\alpha} \phi^{\alpha}\left(p_{a^{\prime}}\right),
\end{aligned}
$$

where $a$ labels the vertex operators for $A_{i}$ and $a^{\prime}$ the ones for $\Phi^{\alpha}$. Since the open Wilson line now involves $\Phi^{\alpha}$ as well as $A_{i}$ there are additional contributions to the surface terms of the $\tau$ integration in the process of making $\theta^{i m} \partial_{m} A_{n} l^{n}$ to $\theta^{i m} F_{m n} l^{n}$. They are precisely necessary terms to make $\theta^{i m} \partial_{m} \Phi^{\alpha} y_{\alpha}$ in the last term of (98) to $\theta^{i m} D_{m} \Phi^{\alpha} y_{\alpha}$ and we also expect that $\theta^{i m} \theta^{j n} \partial_{m} \partial_{n} \Phi^{\alpha} y_{\alpha}$ in (99) becomes $\theta^{i m} \theta^{j n} D_{m} D_{n} \Phi^{\alpha} y_{\alpha}$. After symmetrizing the indices $i$ and $j$, we obtain the expression for $T^{i j}$ (46).

\section{B Conservation of Energy-Momentum Tensor}

We prove

$$
k_{i} T^{i j}+k_{\alpha} T^{\alpha j}=0,
$$


in bosonic string theory. Let us begin with some preparations. It is convenient to define

$$
D_{\tau} \mathcal{O}(x+l \tau) \equiv l^{i} D_{i} \mathcal{O}(x+l \tau)+i\left[y_{\alpha} \Phi^{\alpha}, \mathcal{O}\right](x+l \tau) .
$$

Since

$$
\begin{gathered}
*\left[\exp \left(i \int_{0}^{1} d \tau l^{i} A_{i}(x+l \tau)+y_{\alpha} \Phi^{\alpha}(x+l \tau)\right) \int_{0}^{1} d \tau^{\prime} D_{\tau^{\prime}} \mathcal{O}\left(x+l \tau^{\prime}\right)\right] \\
=*\left[\exp \left(i \int_{0}^{1} d \tau l^{i} A_{i}(x+l \tau)+y_{\alpha} \Phi^{\alpha}(x+l \tau)\right) \mathcal{O}(x+l)\right. \\
\left.-\mathcal{O}(x) \exp \left(i \int_{0}^{1} d \tau l^{i} A_{i}(x+l \tau)+y_{\alpha} \Phi^{\alpha}(x+l \tau)\right)\right],
\end{gathered}
$$

where the path ordering is implicit, we have

$$
\int d x *\left[e^{i k x} \exp \left(i \int_{0}^{1} d \tau l^{i} A_{i}(x+l \tau)+y_{\alpha} \Phi^{\alpha}(x+l \tau)\right) \int_{0}^{1} d \tau^{\prime} D_{\tau^{\prime}} \mathcal{O}\left(x+l \tau^{\prime}\right)\right]=0
$$

The following formula is also useful:

$$
\begin{aligned}
& *\left[\exp \left(i \int_{0}^{1} d \tau l^{i} A_{i}(x+l \tau)+y_{\alpha} \Phi^{\alpha}(x+l \tau)\right) \times\right. \\
& \left.\times \int_{0}^{1} d \tau^{\prime} D_{\tau^{\prime}} f\left(x+l \tau^{\prime}\right) \int_{0}^{1} d \tau^{\prime \prime} g\left(x+l \tau^{\prime \prime}\right)\right] \\
= & *\left[\exp \left(i \int_{0}^{1} d \tau l^{i} A_{i}(x+l \tau)+y_{\alpha} \Phi^{\alpha}(x+l \tau)\right) \times\right. \\
& \left.\times\left\{\int_{0}^{1} d \tau^{\prime}[f, g]\left(x+l \tau^{\prime}\right)+\int_{0}^{1} d \tau^{\prime} g\left(x+l \tau^{\prime}\right) f(x+l)-f(x) \int_{0}^{1} d \tau^{\prime} g\left(x+l \tau^{\prime}\right)\right\}\right] .
\end{aligned}
$$

Now consider

$$
\begin{aligned}
k_{i} T^{i j}= & \frac{\theta^{j n}}{(2 \pi)^{2}} \int d x *\left[e^{i k x} \exp \left(i \int_{0}^{1} d \tau l^{i} A_{i}(x+l \tau)+y_{\alpha} \Phi^{\alpha}(x+l \tau)\right) \times\right. \\
& \times\left\{i \int_{0}^{1} d \tau^{\prime} e^{-2 \pi i \tau^{\prime}} y_{\alpha} D_{\tau^{\prime}} \Phi^{\alpha}\left(x+l \tau^{\prime}\right) \times\right. \\
& \times i \int_{0}^{1} d \tau^{\prime \prime} e^{2 \pi i \tau^{\prime \prime}}\left(l^{n^{\prime}} F_{n n^{\prime}}\left(x+l \tau^{\prime \prime}\right)+y_{\beta} D_{n} \Phi^{\beta}\left(x+l \tau^{\prime \prime}\right)\right)+ \\
& +i \int_{0}^{1} d \tau^{\prime} e^{-2 \pi i \tau^{\prime}}\left(l^{m^{\prime}} F_{n m^{\prime}}\left(x+l \tau^{\prime}\right)+y_{\alpha} D_{n} \Phi^{\alpha}\left(x+l \tau^{\prime}\right)\right) \times \\
& \times i \int_{0}^{1} d \tau^{\prime \prime} e^{2 \pi i \tau^{\prime \prime}} y_{\beta} D_{\tau^{\prime \prime}} \Phi^{\beta}\left(x+l \tau^{\prime \prime}\right)+
\end{aligned}
$$




$$
\begin{aligned}
& +i \int_{0}^{1} d \tau^{\prime}\left(l^{n^{\prime}} l^{m} D_{m} F_{n n^{\prime}}\left(x+l \tau^{\prime}\right)\right. \\
& \left.\left.\left.+y_{\alpha} l^{m} D_{m} D_{n} \Phi^{\alpha}\left(x+l \tau^{\prime}\right)+y_{\alpha} l^{m} D_{n} D_{m} \Phi^{\alpha}\left(x+l \tau^{\prime}\right)\right)\right\}\right],
\end{aligned}
$$

where we have used that

$$
y_{\alpha} l^{m} D_{m} \Phi^{\alpha}(x+l \tau)=y_{\alpha} D_{\tau} \Phi^{\alpha}(x+l \tau) .
$$

There are two different contributions when we make partial integrations with respect to $D_{\tau^{\prime}}$ in the second line and $D_{\tau^{\prime \prime}}$ in the fifth line. The first one is the one which arises when $D_{\tau^{\prime}}\left(D_{\tau^{\prime \prime}}\right)$ acts on $e^{-2 \pi i \tau^{\prime}}\left(e^{2 \pi i \tau^{\prime \prime}}\right)$ and this is precisely canceled by $k_{\alpha} T^{\alpha j}$. The other one can be evaluated using the formula (104) and gives a factor

$$
2 i^{2} \int_{0}^{1} d \tau^{\prime}\left[y_{\alpha} \Phi^{\alpha}, l^{n^{\prime}} F_{n n^{\prime}}+y_{\beta} D_{n} \Phi^{\beta}\right]
$$

The three terms in the last two lines of (105) can be written as

$$
\begin{aligned}
l^{n^{\prime}} l^{m} D_{m} F_{n n^{\prime}}\left(x+l \tau^{\prime}\right) & =l^{n^{\prime}} D_{\tau^{\prime}} F_{n n^{\prime}}\left(x+l \tau^{\prime}\right)-i\left[y_{\alpha} \Phi^{\alpha}, l^{n^{\prime}} F_{n n^{\prime}}\right]\left(x+l \tau^{\prime}\right), \\
y_{\alpha} l^{m} D_{m} D_{n} \Phi^{\alpha}\left(x+l \tau^{\prime}\right) & =y_{\alpha} D_{\tau^{\prime}} D_{n} \Phi^{\alpha}\left(x+l \tau^{\prime}\right)-i\left[y_{\beta} \Phi^{\beta}, y_{\alpha} D_{n} \Phi^{\alpha}\right]\left(x+l \tau^{\prime}\right), \\
y_{\alpha} l^{m} D_{n} D_{m} \Phi^{\alpha}\left(x+l \tau^{\prime}\right) & =y_{\alpha} l^{m} D_{m} D_{n} \Phi^{\alpha}\left(x+l \tau^{\prime}\right)+i\left[l^{m} F_{n m}, y_{\alpha} \Phi^{\alpha}\right]\left(x+l \tau^{\prime}\right),
\end{aligned}
$$

so that the last integral of (105) gives

$$
i \int_{0}^{1} d \tau^{\prime}\left(-2 i\left[y_{\alpha} \Phi^{\alpha}, l^{n^{\prime}} F_{n n^{\prime}}\right]-2 i\left[y_{\beta} \Phi^{\beta}, y_{\alpha} D_{n} \Phi^{\alpha}\right]\right)
$$

which cancels (107). Thus we have shown the conservation (100). 


\section{References}

[1] A. Connes, M. R. Douglas, and A. Schwarz, "Noncommutative geometry and matrix theory: Compactification on tori," JHEP 02 (1998) 003, hep-th/9711162.

[2] M. R. Douglas and C. Hull, "D-branes and the noncommutative torus," JHEP 02 (1998) 008, hep-th/9711165.

[3] Y.-K. E. Cheung and M. Krogh, "Noncommutative geometry from 0-branes in a background B-field," Nucl. Phys. B528 (1998) 185-196, hep-th/9803031.

[4] T. Kawano and K. Okuyama, "Matrix theory on noncommutative torus," Phys. Lett. B433 (1998) 29-34, hep-th/9803044.

[5] F. Ardalan, H. Arfaei, and M. M. Sheikh-Jabbari, "Noncommutative geometry from strings and branes," JHEP 02 (1999) 016, hep-th/9810072.

[6] C.-S. Chu and P.-M. Ho, "Noncommutative open string and D-brane," Nucl. Phys. B550 (1999) 151-168, hep-th/9812219.

[7] V. Schomerus, "D-branes and deformation quantization," JHEP 06 (1999) 030, hep-th/9903205.

[8] F. Ardalan, H. Arfaei, and M. M. Sheikh-Jabbari, "Dirac quantization of open strings and noncommutativity in branes," Nucl. Phys. B576 (2000) 578-596, hep-th/9906161.

[9] C.-S. Chu and P.-M. Ho, "Constrained quantization of open string in background B field and noncommutative D-brane," Nucl. Phys. B568 (2000) 447-456, hep-th/9906192.

[10] N. Seiberg and E. Witten, "String theory and noncommutative geometry," JHEP 09 (1999) 032, hep-th/9908142.

[11] Z. Yin, "A note on space noncommutativity," Phys. Lett. B466 (1999) 234-238, hep-th/9908152.

[12] S. R. Das and S.-J. Rey, "Open Wilson lines in noncommutative gauge theory and tomography of holographic dual supergravity," Nucl. Phys. B590 (2000) 453-470, hep-th/0008042.

[13] D. J. Gross, A. Hashimoto, and N. Itzhaki, "Observables of non-commutative gauge theories," hep-th/0008075. 
[14] N. Ishibashi, S. Iso, H. Kawai, and Y. Kitazawa, "Wilson loops in noncommutative Yang-Mills," Nucl. Phys. B573 (2000) 573-593, hep-th/9910004.

[15] J. Ambjorn, Y. M. Makeenko, J. Nishimura, and R. J. Szabo, "Finite N matrix models of noncommutative gauge theory," JHEP 11 (1999) 029, hep-th/9911041.

[16] J. Ambjorn, Y. M. Makeenko, J. Nishimura, and R. J. Szabo, "Lattice gauge fields and discrete noncommutative Yang-Mills theory," JHEP 05 (2000) 023, hep-th/0004147.

[17] A. Dhar and S. R. Wadia, "A note on gauge invariant operators in noncommutative gauge theories and the matrix model," hep-th/0008144.

[18] A. Dhar and Y. Kitazawa, "Wilson loops in strongly coupled noncommutative gauge theories," hep-th/0010256.

[19] A. Dhar and Y. Kitazawa, "High Energy Behavior of Wilson Lines," hep-th/0012170.

[20] S. Hyun, Y. Kiem, S. Lee, and C.-Y. Lee, "Closed strings interacting with noncommutative D-branes," Nucl. Phys. B569 (2000) 262-276, hep-th/9909059.

[21] M. R. Garousi, "Non-commutative world-volume interactions on D-branes and Dirac-Born-Infeld action," Nucl. Phys. B579 (2000) 209-228, hep-th/9909214.

[22] H. Liu and J. Michelson, "*-TREK: The one loop $\mathrm{N}=4$ noncommutative SYM action," hep-th/0008205.

[23] D. Zanon, "Noncommutative perturbation in superspace," hep-th/0009196.

[24] A. Santambrogio and D. Zanon, "One-loop four-point function in noncommutative N=4 Yang-Mills theory," hep-th/0010275.

[25] T. Mehen and M. B. Wise, "Generalized *-products, Wilson lines and the solution of the Seiberg-Witten equations," hep-th/0010204.

[26] H. Liu, “*-Trek II: $*_{n}$ operations, open Wilson lines and the Seiberg-Witten map," hep-th/0011125.

[27] S. R. Das and S. P. Trivedi, "Supergravity couplings to noncommutative branes, open Wilson lines and generalized star products," hep-th/0011131. 
[28] M. Pernici, A. Santambrogio, and D. Zanon, "The one-loop effective action of noncommutative $\mathrm{N}=4$ super Yang-Mills is gauge invariant," hep-th/0011140.

[29] M. R. Garousi, "Transformation of the Dirac-Born-Infeld action under the Seiberg-Witten map," hep-th/0011147.

[30] Y. Kiem, D. H. Park, and S. Lee, "Factorization and generalized *-products," hep-th/0011233.

[31] A. Hashimoto and N. Itzhaki, "Non-commutative Yang-Mills and the AdS/CFT correspondence," Phys. Lett. B465 (1999) 142-147, hep-th/9907166.

[32] J. M. Maldacena and J. G. Russo, "Large N limit of non-commutative gauge theories," JHEP 09 (1999) 025, hep-th/9908134.

[33] E. S. Fradkin and A. A. Tseytlin, "Nonlinear electrodynamics from quantized strings," Phys. Lett. B163 (1985) 123.

[34] C. G. Callan, C. Lovelace, C. R. Nappi, and S. A. Yost, "String loop corrections to beta functions," Nucl. Phys. B288 (1987) 525.

[35] A. Abouelsaood, C. G. Callan, C. R. Nappi, and S. A. Yost, "Open strings in background gauge fields," Nucl. Phys. B280 (1987) 599.

[36] O. Andreev and H. Dorn, "Diagrams of noncommutative $\Phi^{3}$ theory from string theory," Nucl. Phys. B583 (2000) 145-158, hep-th/0003113.

[37] A. Bilal, C.-S. Chu, and R. Russo, "String theory and noncommutative field theories at one loop," Nucl. Phys. B582 (2000) 65-94, hep-th/0003180.

[38] J. Gomis, M. Kleban, T. Mehen, M. Rangamani, and S. Shenker, "Noncommutative gauge dynamics from the string worldsheet," JHEP 08 (2000) 011, hep-th/0003215.

[39] J. Maldacena, "The large N limit of superconformal field theories and supergravity," Adv. Theor. Math. Phys. 2 (1998) 231-252, hep-th/9711200.

[40] M. Alishahiha, Y. Oz, and M. M. Sheikh-Jabbari, "Supergravity and large N noncommutative field theories," JHEP 11 (1999) 007, hep-th/9909215.

[41] M. Rozali and M. V. Raamsdonk, "Gauge invariant correlators in non-commutative gauge theory," hep-th/0012065. 
[42] S. S. Gubser, I. R. Klebanov, and A. M. Polyakov, "Gauge theory correlators from non-critical string theory," Phys. Lett. B428 (1998) 105-114, hep-th/9802109.

[43] E. Witten, "Anti-de Sitter space and holography," Adv. Theor. Math. Phys. 2 (1998) 253-291, hep-th/9802150.

[44] S.-J. Rey and J. Yee, "Macroscopic strings as heavy quarks in large N gauge theory and anti-de Sitter supergravity," hep-th/9803001.

[45] J. Maldacena, "Wilson loops in large N field theories," Phys. Rev. Lett. 80 (1998) 4859-4862, hep-th/9803002.

[46] N. Drukker, D. J. Gross, and H. Ooguri, "Wilson loops and minimal surfaces," Phys. Rev. D60 (1999) 125006, hep-th/9904191. 